\title{
DATA ACQUISITION ARCHITECTURE FOR CAR SIMULATORS: APPLICATION IN DRIVING ADAPTATIONS FOR DISABLED
}

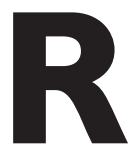

oad safety is a major public health problem in developed countries. Each year, around 1.2 million people die in the road traffic system (around 3000 per day) and more than 50 million are injured worldwide. About $2 \%$ of all deaths from any cause are related to traffic injuries, and about $23 \%$ of all injury deaths are caused by traffic accidents. Globally, there is a factor of around 40 between the number of injuries and the number of fatalities. The European Council of Ministers of Transport (ECMT) in Bucharest in 2002 unanimously adopted a common quantitative target for all member countries to reduce the 2000 road fatality level by $50 \%$ by 2012. Similarly, the European Commission agreed on a target of 50\% reduction in the number of road fatalities by the year 2010, compared with 2001. The social and human costs of all these road crashes are about US $\$ 518$ billion every year. For this reason, the United Nations (UN) and the World Health Organization (WHO) pleads for effective and sustainable road traffic safety policies. Many countries give high priority to road traffic safety, with ambitious targets set for the upcoming years. ${ }^{1}$ Road traffic accidents in the Member States of the European Union annually claim about 43,000 lives and leave more than 1.8 million people injured, representing estimated costs of 160 billion euros. ${ }^{2}$

However, between 10 and $14 \%$ of the population in many nations is disabled or suffers limited mobility due to physical, mental, or sensorial problems-according to the ECMT. This means some 50 million people in the ECMT member countries cannot move freely from one place to another. ${ }^{3}$

According to a survey on Disability, Deficiency, and Health, conducted by the Spanish National Institute of Statistics (INE) ${ }^{4}$ the total number of people in Spain with permanent disabilities resulting from physical, sensory, or mental injuries or diseases amounts to 3,528,221, nearly $9 \%$ of the total population. Data from the Spanish National Traffic Administration (DGT) from the year 2003 showed that around 120,000 disabled drivers had adaptations in their vehicles, although the estimated number of drivers who need them is greater. ${ }^{5}$ Moreover, it must be mentioned that more than half of the drivers with disabilities disagree with the adaptations recommended by the traffic administration for obtaining a driving license because these adaptations do not match their actual needs for driving.

This is why one of the main problems for a disabled driver is to select the control adaptations that best match his/her disability. The disabled driver also has the problem of showing objectively that he/she is able to drive in

J.C. Campelo is an associate professor, A. Martí is an associate professor, J. Pardo (juaparal@uch.ceu.es) is an associate professor, and J.J. Serrano is a full professor with the Department of Computer Engineering, Technical University of Valencia, Valencia, Spain. a complete safety way. Often the traffic administration must make decisions which should be based on technical criteria, and yet are actually based on experience and subjective evaluations of a driver's residual capacity. Other times, an adaptation is chosen by technical installers who select the recommended type of adaptation from the range offered by their own preferred manufacturers. These factors mean that two identical disabled drivers might hear contradictory answers and receive totally different adaptations. This situation makes it necessary to establish technical guidelines, legislation, and tools for evaluating the driving abilities of each driver.

Some of the basic problems suffered by disabled people when driving adapted vehicles ${ }^{6}$ are the utilization of primary controls (accelerator, brakes, and steering wheel) and secondary controls (indicators, horn, windows, lights, windshield wipers, etc). The selection of the best vehicle control adaptation allows the disabled driver drive with the same level of safety and comfort as an able-bodied driver. To achieve this, the disabled driver must reach all the controls necessary to control the vehicle with enough strength to activate them and be able to react quickly enough in an emergency situation. This is the objective of the research program carried out at the Automobile Laboratory of the Department of Mechanical Engineering and Materials of the Technical University of Valencia (Spain). Since 1995, they have been involved in the design of several simulators-evaluators to objectively study the abilities of any disabled driver to demonstrate which type of adaptation is more appropriate to the driver. The simulator-evaluator reproduces the driving position, as well as the driving environment, with dynamic traffic scenes and situations that the driver usually experiences. However, to perform these kinds of studies, the data acquisition system of the driving simulator has to be able to obtain, with high accuracy, all the relevant measurements to accomplish with the evaluation and training needed requirements. However, the problem is that data acquisition systems of actual car simulators (and also the previous versions of the simulator developed by the Technical University of Valencia) have two important constraints: limited sampling rate and non real-time behavior to carry out such studies.

Centralized data acquisition systems based on old microprocessors make it impossible to sample a lot of magnitudes, process them, and communicate with the video projection subsystem in a deterministic way and with a realistic sensation for the driver under evaluation. Hence, only when a few magnitudes are sampled simple serial connections and standard processors can be enough, but when the simulator tries to simulate the real-world sampling all magnitudes involved in the dynamic process of driving, powerful real-time distributed systems based on industrial local area networks (field buses) are necessary. Thus, it is necessary to develop 
a real-time distributed architecture that supports actual requirements.

\section{THE CAR SIMULATOR}

Traditionally, driver training and evaluation has focused on vehicle control skills and traffic rules without reaching far enough in the efforts to provide risk awareness and other higher order skills to improve the driving safety. The risk awareness problem is included in driver training in many countries but rather in a theoretical way and it is not covered in practical training. ${ }^{7}$ The reason is that this problem cannot be systematically handled in practical training, as on roads it is very uncertain and even dangerous that a risk situation may occur. According to Reed and Green, ${ }^{8}$ there are three primary justifications for using driving simulation rather than in-vehicle testing: (1) Safety: Some research is too hazardous to be conducted in vehicles on the road (e.g., studies of collision avoidance, reaction time on maneuvers, etc). Moreover, it is safer to study multiple-vehicle scenarios in a simulator than on an actual road. (2) Equipment cost: Simulators allow study of driver responses to changes in the vehicle without having to construct a vehicle with those features or performance characteristics. (3) Experimental control: a wider variety of test conditions can be prescribed and consistently applied in a driving simulator than on the road. For example, the influence of the weather on driving conditions is controllable in the simulator.

These are some of the main benefits of using driving simulators to train and evaluate future drivers before going to the real roads, and mainly with disabled drivers where the configuration of the driving elements inside the car should be checked enough for a safer driving. The main characteristics of the simulator at the Technical University of Valencia are ${ }^{7}$ :

\section{Body/Structure}

The cockpit is open (i.e., without doors) to facilitate the observation of the driver behavior, and the type of vehicle emulated is a Ford Focus. All the mandatory elements on the dashboard work as in reality and the software registers their activations.

\section{Pedals and Driving Seat}

The pedals are the original accelerator, brake, and clutch of a Ford Focus. The brake and clutch pedals have a force sensor of $500 \mathrm{~N}$ to measure the force applied to them. It is also possible to measure angles of the pedals and reaction time using linear differential variable transformers (LDVTs). The driving seat is a standard mechanical driving seat with three degrees of freedom (horizontal, height, and inclination).

\section{Steering Wheel Device}

The steering wheel is the original one, attached to a DC $12 \mathrm{v}$ motor with a gear head which produces a reduction of 70/4, producing a maximum torque of $6 \mathrm{~N}$ controlled by current. A string potentiometer is included to know the position and movements of the steering wheel. It is also adjustable to improve the comfort of the disabled, and there is a vibration on the steering at running vehicle engine, produced by an off-centered motor connected to the shaft of the steering wheel.

\section{Gearbox and Handbrake Levers}

Gear stick, gearbox, and handbrake lever are also the original of a car. These produce digital signal for the data acquisition card, and it is possible to drive with automatic or manual transmission.

\section{Motion System}

The simulator has an electrical motion system included with six degrees of freedom, simulating movements of roll, pitch, and yaw, also simulating lateral transient forces in the horizontal direction by means of electrical motors. The motion system is self-controlled, and the simulator calculates and sends to this motion system the magnitude and direction of the forces to be simulated.

\section{Visual System}

For the visual system, three video projectors are used to obtain a field of view close to $180^{\circ}$. There are rear view mirrors, created by the simulation software, and the image is computer generated.

\section{Audio System}

Visual feedback has always a predominating position within the means used to generate realistic surroundings in a driving simulator. However, the contribution of an acoustic feedback combining the representation of sound sources from inside and outside the vehicle improves the realism of the driving situation. Sounds are reproduced using a Pc-soundcard with an audio amplifier and loudspeakers of $40 \mathrm{~W}$.

\section{Computer System}

The computer system is based on Obsidian FX2 from the company Quantum3D (San Jose, CA); it is a commercial of-the-self image generator platform for 30 and $60 \mathrm{~Hz}$ midrange, multichannel synthetic environment simulation. With this equipment, it is possible to synchronize via hardware, with the Quantum3D nVsync ${ }^{\circledR}$ device, different visual channels keeping the image generation fresh rate, which is a typical problem of driving simulators.

\section{Software Characteristics}

The simulation software has a user-friendly interface with a lot of pull-down menus, icons, sounds, etc, and it has been developed in $\mathrm{C}++$, Vega, and Multigen Creator. The software has a training program with a lot of different scenarios in which the user can save his/her skill on passed lessons and to evaluate his/her progress on the training. The selection of the different scenarios comes from a European project named TRAINER, in which the Automobile Laboratory participated, which ranges a lot of different real situations related to the driving safety. The software includes statistics on driving faults, on passed lessons, statistics of simulator use, etc. The sight conditions are rain, fog, snow, and night. On the screen, the user finds different messages and alerts. All kinds of scenarios to measure the reaction time are available. There is also a previous static simulation in which the forces, angles, and other different variables are measured to study which adaptation will be better for the later dynamic simulation. 


\section{REAL-TIME DATA ACQUISITION SYSTEM DESIGN}

In order to define the acquisition system architecture, the next requirements have been taken into account:

(1) Scalability and versatility: It will be necessary to install different types of adaptations during the individual studies. This means that the digitalization process must be suitable for the existing sensors, as well as being versatile enough to handle future larger configurations. (2) Sampling rate and real-time: the data acquisition system must acquire, communicate, and actuate in a deterministic manner and with a maximum and known delay.

\section{System Overview}

The data acquisition system is designed as a distributed industrial control system. Hence, several nodes are distributed around the simulator to acquire measures, calculate the control actions, and actuate over different simulator subsystems. Basically, two different nodes can be distinguished: digital and analog. First ones are those in charge of control digital signals and second ones are in charge of control analog signals. When dealing with analog signals, three types of sensors are commonly used: LVDTs, potentiometers, and load cells. These sensors measure position or pressure of the driver over some lever, pedal, or steering wheel. On the other side, digital inputs inform about the activation of some control of the cockpit. Communications between the nodes of the system is accomplished by using a Controller Area Network (CAN) network. ${ }^{9}$

In Fig. 1, the system is outlined. In this figure, different nodes can be observed: (1) Clutch pedal node: This node informs about the clutch pedal activation and the driver pressure on it. (In the case of adaptations certification, it is very important to be sure about the ability of a driver to push a pedal with enough force). (2) Break pedal node: As the previous one, this node informs about the break pedal activation and the driver pressure. (3) Throttle pedal node: This node informs about the position of the throttle pedal. (4) Adaptations node: It is a generic node to be connected to a specific adaptation (i.e., an extension of the pedals to be moved by hands, etc). (5) Gear lever and control panel node: This node informs about the gear box selection and the controls of the cockpit. (6) Steering-wheel node: This node informs about the position of the steering wheel and the strength applied by the driver.

\section{Microcontroller-Based Architecture}

Over the years, the increasing availability of high-speed and low-cost microcontrollers has encouraged the use of such devices for a variety of applications, including realtime control. Microcontrollers are very usual components of modern electronic systems. They are in charge of executing the control algorithms and supervising systems behavior. From a microcontroller core, manufacturers develop specific versions, adding different peripheral devices in the integrated circuit, to satisfy different application areas such as automotive, mobile telephony, security systems, industrial systems, appliances, etc. Microcontrollers can be classified in 8-, 16-, and 32-bit families. The simplest (8-bit microcontrollers) are those that present a low computational power and a low price, so they are a very interesting option to control many of systems. However, 16- and 32-bit microcontrollers offer an improved performance but at a higher cost. Nevertheless, many microcontrollers' manufacturers are developing new versions of the classical 8-bit microcontrollers with a high computational power, so they are a very interesting option in front of the more expensive 16-bit microcontrollers. This way, different manufacturers develop from the basic 8051-architecture ${ }^{10,11}$ new models with more on-chip peripherals such as timers, capture-compare units, watchdog timer, pulse-width modulation (PWM) outputs, several communication channels (universal asynchronous receiver-transmitters [UARTs], $\mathrm{CAN}$, serial peripheral interface [SPI], inter-integrated circuit $\left[\mathrm{I}^{2} \mathrm{C}\right]$, universal serial bus [USB], etc), $\mathrm{AD}$ converter, internal flash memory, and a high computational (up to 100 MIPS). ${ }^{11}$

The distributed industrial control system designed is composed by nodes based on the last generation 8-bit microcontrollers, and it is implemented in a modular way. Therefore, nodes are composed of different boards according to their objectives. As it had been said before, two different kinds of nodes can be distinguished, one devoted to digital signals processing and another for analog signals. Nodes are composed of two boards; one of them has, basically, the microcontroller, network connections, and connectors to connect its buses with another board. The other board has the circuitry to process the digital or analog signals. Figure 2 shows a photograph of the first board: the microcontroller, two big connectors (at borders) to connect another board to the microcontroller buses, two CAN connectors at the bottom side and (in the top side) power, and Joint Test Action Group (JTAG) connector to program the microcontroller.

The microcontroller used for the execution of control algorithms is the SILABS C8051F041 (Silicon Labs, Austin, TX). ${ }^{11}$ System overview from the C8051F04x microcontroller family manual ${ }^{11}$ : The C8051F04x family of devices are fully integrated mixed-signal System-on-a-Chip microcontroller units (MCUs) with 64 digital I/O pins (C8051F040/2) or 32 digital I/O pins (C8051F041/3), and an integrated CAN 2.0B controller.

\section{Analog Module}

The analog module is based on the described microcontroller and the necessary circuitry to prepare the signals to be converted to digital signals. Each analog node must be able to read two sensors (position and force), apply a calibration curve, and send data through the network. There are three different kinds of sensors in the system: load cell to measure driver's force on pedals, with an output signal lower than $20 \mathrm{mV}$ for full-scale input; LVDT used to measure pedal position, presenting bipolar output ranging from -3 to $+3 \mathrm{~V}$; and string potentiometer attached to steering wheel to measure its position, with unipolar output up to $5 \mathrm{~V}$. The signal conditioner circuit must prepare these outputs to microcontroller converter, which only accept 


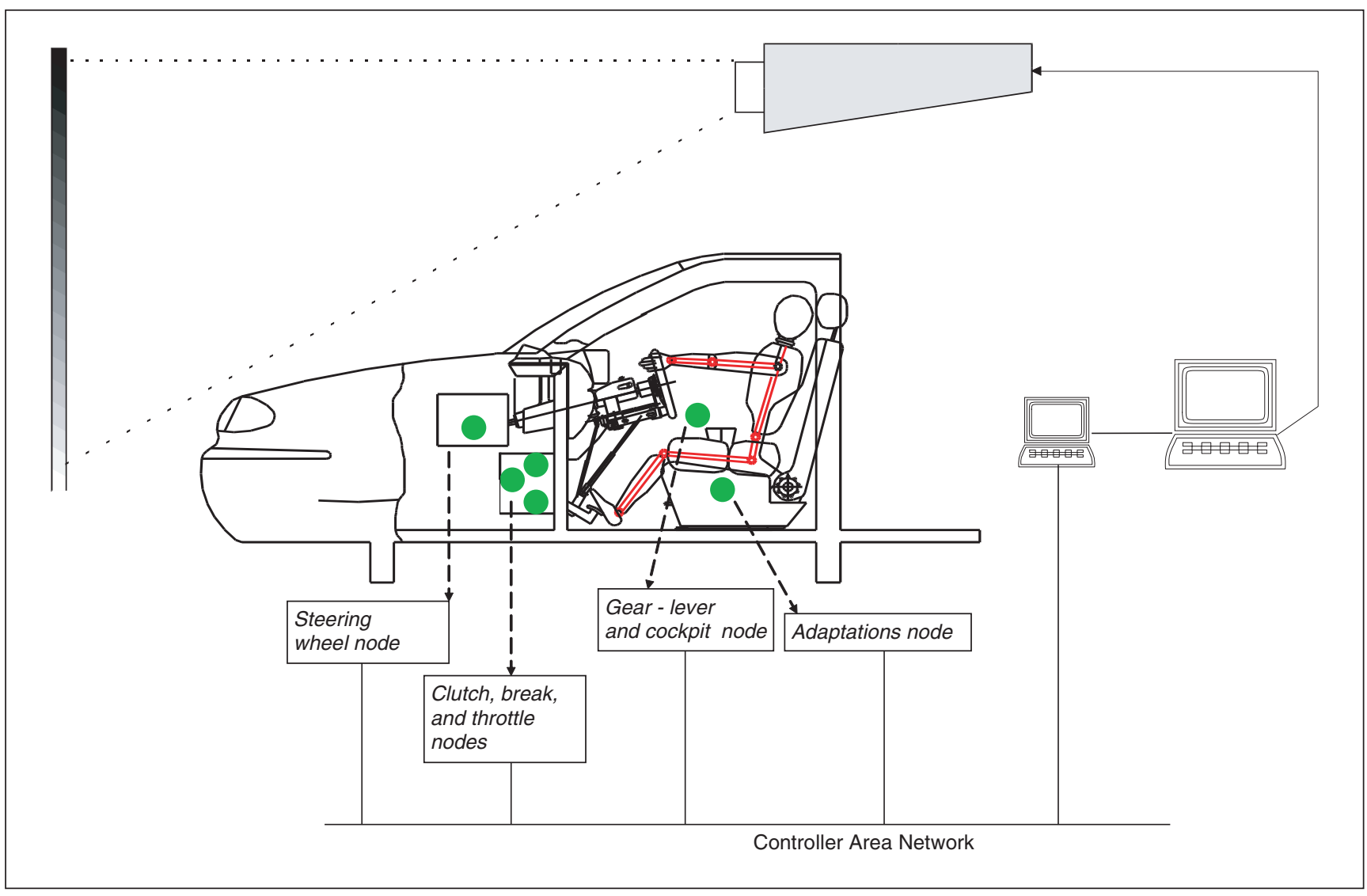

Fig. I: System overview

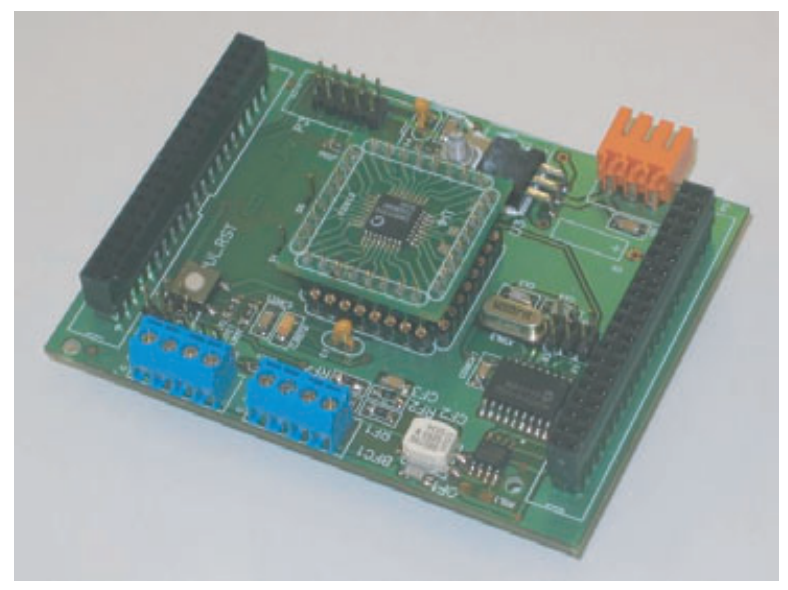

Fig. 2: Photograph of the microcontroller board

positive voltages in the range $0-3.6 \mathrm{~V}$. Signal conditioner circuit must be able to work with other, future-use sensors. Figure 3 shows the block diagram of signal conditioner circuit.

Output from the signal conditioner circuit is connected to the microcontroller built-in high voltage difference amplifier (HVDA). This HVDA can be used to measure high differential voltages up to $60 \mathrm{~V}$ peak-to-peak, reject high common-mode

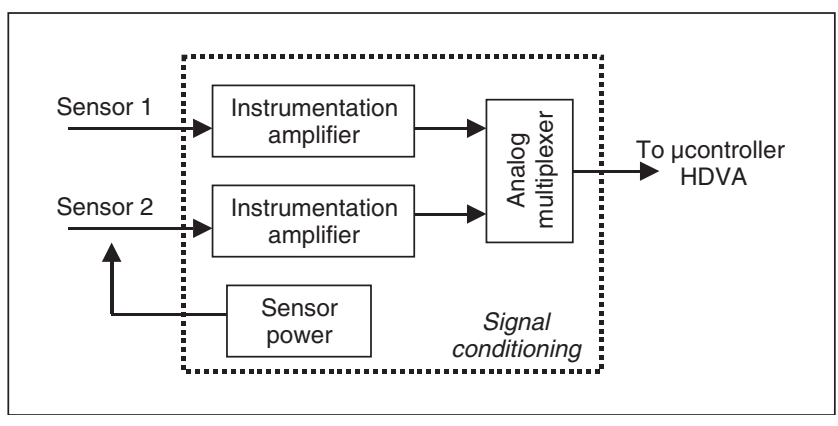

Fig. 3: Signal conditioner circuit

voltages up to $\pm 60 \mathrm{~V}$, and condition the signal voltage range to be suitable for input to analog-to-digital converter (ADC) converter. The input signal to the HVDA may be below analog ground (AGND) to $-60 \mathrm{~V}$, and as high as $+60 \mathrm{~V}$, making the device suitable for both single and dual supply applications. The HVDA will provide a common-mode signal for the ADC via the high voltage reference input (HVREF), allowing measurement of signals outside the specified ADC input range using on-chip circuitry. The HVDA has a gain of 0.05 to $14 \mathrm{~V} / \mathrm{V}$. The HVDA is factory calibrated for a high common-mode rejection of $72 \mathrm{~dB}$. The use of HVDA allows connecting a large variety of sensors with no modification to hardware. However, HDVA presents two shortcomings. 


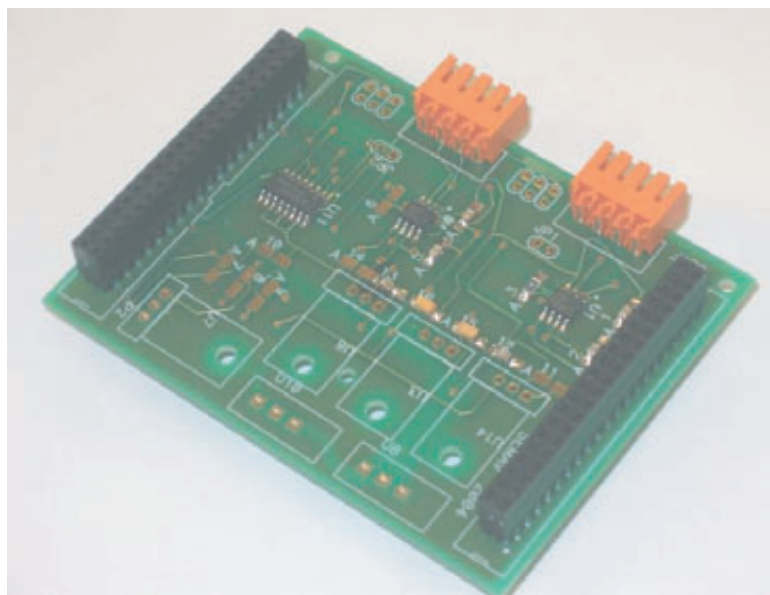

Fig. 4: Photograph of the analog input board

First, its input impedance is too low for potentiometerbased sensor, and second, its gain is too low for load cell sensors. To match impedances and to increase the gain, an INA121/122 $2^{12,13}$ instrumentation amplifier is used to prepare input signals to the HVDA.

The INA121 (similar for INA122) is a field-effect transistor (FET), high impedance input, low-power instrumentation amplifier offering excellent accuracy. Its versatile threeoperational amplifiers design and very small size make it ideal for a variety of general purpose applications. Low bias current $( \pm 4 \mathrm{pA})$ allows use with high impedance sources. Gain can be set from 1 to 10,000 V/V with a single external resistor. Internal input protection can withstand up to $\pm 40 \mathrm{~V}$ without damage. The INA121 is laser-trimmed for very low offset voltage $( \pm 200 \mathrm{mV})$, low offset drift $\left( \pm 2 \mathrm{mV} /{ }^{\circ} \mathrm{C}\right)$, and high common-mode rejection $(106 \mathrm{~dB}$ at $G=100)$. It operates on power supplies as low as $\pm 2.25 \mathrm{~V}(+4.5 \mathrm{~V})$, allowing use in battery-operated and single $5 \mathrm{~V}$ systems. Quiescent current is only $450 \mathrm{~mA}$. Package options include 8-pin plastic DIP and SO-8 surface mount. All are specified for the -40 to $+85^{\circ} \mathrm{C}$ industrial range. Finally, a multiplexer is also used to sample sequentially the outputs from the two instrumentation amplifiers (INAs) and to route them to the HDVA temperature range. The signal conditioner circuitry constitutes a board that is stacked to the microcontroller's board previously described. This board can be seen in Fig. 4 (the two INAs and the multiplexer can be seen). Moreover, other components like voltage regulators (7805, 7815,7812 - not soldered in the photograph of Fig. 4) are necessary to generate the necessary voltage levels to INA amplifiers.

The main software of analog node (the union of the microcontroller and the signal conditioning board) is a time-driven loop that reads sensor outputs and sends them through the network. The acquisition procedure is interrupt activated by the overflow of a general-purpose timer included in the microcontroller. The count of this timer is configured from the network. Once the procedure is activated, three samples are obtained from sensor one, discarding the two extreme values. From sensor two, three samples are also obtained, and only the median (center value) is kept. Next, a second-order calibration curve is applied to both median values, and results are sent to the simulator node. Calibration curves are defined separately for each sensor, and its coefficients are received from the network. The use of median values, in front of single sample or averaging samples, allows minimizing interference effects and transient errors along the sensor-to-digital chain.

Real-time requirement of analog node has been verified by means of static analysis. The microcontroller does not present hardware improvements, such as cache memory, branch prediction, or speculative execution, so execution of instructions is constant and its worst case execution time may be estimated using simple timing analysis. ${ }^{14}$ Analog-todigital conversion time is safely bounded by microcontroller manufacturer, adding this time to code execution time. Finally, network management is also bounded, and time to send data is included in acquisition loop. Static analysis, experimentally corroborated, shows a maximum samplingand-sending frequency of $800 \mathrm{~Hz}$.

\section{Sensors Calibration}

One of the main objectives of this distributed system is to get the maximum performance from the acquisition system. That is, reach the maximum sampling frequency possible. The software that has to evaluate and simulate the driver's actions needs to work with physical quantities, like displacement or torque. Hence, the values collected from sensors have to be converted into these quantities. If the main computer receives values in volts and amperes, it has to do hard work to convert them, so it is possible that the computational time required to make conversions limits the maximum sampling frequency. This way, the translation from the output values of sensors and useful values for the simulator is accomplished in the microcontroller-based nodes in the network.

Also, it is important to note that sensors are attached in different ways to the mechanical devices, so the theoretical output may be skewed or incorporate some offset.

First, there is an isolated application that allows connecting with each node in the network and sending it a set of parameters to configure the calibration curve for each analog input/output. All the calibration curves are second order. This application is running before the simulator is used. The parameters sent to the nodes are stored in an internal flash memory - no volatile-of the microcontroller, so every time the whole system is powered on, every microcontroller read from this memory the parameters for its sensor. This way, the calibration software has to run only once.

Every sensor needs a different process for its calibration. Following, a calibration process for each type of device used in the system.

- LDVT: This kind of sensor produces a linear output, so its calibration is very easy. However, in this case, the calibration is still easier, because they are used for measuring pedal position. The simulator needs only the percentage of the pedal displacement as the 
driver press or release the pedal. First, the calibration starts setting the calibration curve to read the raw data from the sensor, that is, the output in volts. This output is measured with the pedal in the highest position-released-, and later, measured with the pedal in his lowest position-pressing the pedal up to the end of the run. With only two values-the sensor is linear-and simply mathematical operations, the system operator is able to send the parameter to the microcontroller and get from it the position of the pedal in percentage.

- Load cell: These sensors are attached to the pedal to measure the force applied by the driver. The procedure to calibrate these sensors includes the use of three weights, with nominal values of 1,5 , and $10 \mathrm{~kg}$. Each of the weights is left on the pedal and the output of the load cell is measured in volts using the application for calibration. With the four values - the three weights and a measurement without pressure-the system operator sends the parameters of the calibration curve to the microcontroller.

- String potentiometer: The string potentiometer is used in a voltage divider configuration, and it determines the maximum number of turns the steering wheel can make. Three positions are measured: maximum turn to the left, maximum turn to the right, and center position of the steering wheel. Again, the initial calibration curve is configured to read the raw output of the potentiometer. With the collected values, a new calibration curve is set in the microcontroller to obtain the position of the steering wheel in bipolar percentage-negative values indicate turn to the left.

- DC motor: The DC motor that controls the resistance the steering wheel presents to the driver also has to be calibrated. The previous weights are again used, attaching them to the steering wheel. The system operator sends the microcontroller several values of current to apply to the DC motor. When the steering wheel is stopped in a balance position with the weight at $45^{\circ}$, the current value is recorded and used to make the output calibration curve.

- Time calibration: In order to get a right simulation, not only must the values collected from sensors be correct and accurate, but also the time they are collected has to be coherent. The system operator, during the initial configuration of the simulator, decides the sampling frequency for inputs and outputs, and each node uses its own clock system to reach the sampling frequency. Using high accuracy crystal to keep at minimum the deviation between clock nodes is too expensive and does not guarantee success. Instead of a hardware solution, a network solution is chosen. During the initial installation of the distributed nodes, the frequency of each node has been checked. Once the node with highest accuracy is detected, this node is selected to broadcast every $8 \mathrm{~s}$ a message of synchronization. This message is received by all the nodes in the network, and all of them reset its clock system-without disturbing the acquisition or work in progress-to synchronize the start of the period sample. This way, the deviation between nodes clocks is kept in safe bounds and data from all sensors are collected in a small time window. Also, in any moment, using the same application to set calibration curves, the system operator may select another node to broadcast the synchronization message.

Several adaptations are used to evaluate disabled drivers. Most of these adaptations are mechanical devices that act on the original car elements - for example, a hand-operated brake that transforms hand movement in displacement of the break pedal. In these cases, no re-calibration is needed.

\section{Digital Input/Output Module}

The digital input/output module is based, as the previous one, on the described microcontroller board and the necessary circuitry to read and generate digital 0/1 signals. As the microcontroller works with $3.3 \mathrm{~V}$ and the simulator uses $12 \mathrm{~V}$, the voltage levels need to be adapted. Moreover, the microcontroller ports should be protected against voltage bursts or erroneous connections. For this purpose, relays and optocouplers were considered. The advantages of relays are the higher currents and voltages that can be switched as the output current does not depend on the input current. Optocouplers have the advantages of requiring smaller currents to "switch," they are smaller, and they do not have mechanical parts and therefore they do not wear out. As the outputs should only drive LEDs and the voltage is $12 \mathrm{~V}$, the advantages of the relays are not so important. An optocoupler with a good CTR-current transfer ratio (equal or bigger than 100\%) - can switch the necessary current. However, the optocoupler's advantages like the smaller size and the lesser currents to actuate the switch are quite important as the size of the board is limited and the microcontroller ports are limited in current supply.

To achieve these requirements, a board devoted to adapt voltage levels and to isolate signals is implemented. Figure 5 shows this board. Besides the border connectors (to connect with the microcontroller buses), the board is composed of surface mounted device (SMD) optocouplers and the input/output connectors (PE, PE1, PE2, and PE3 in the

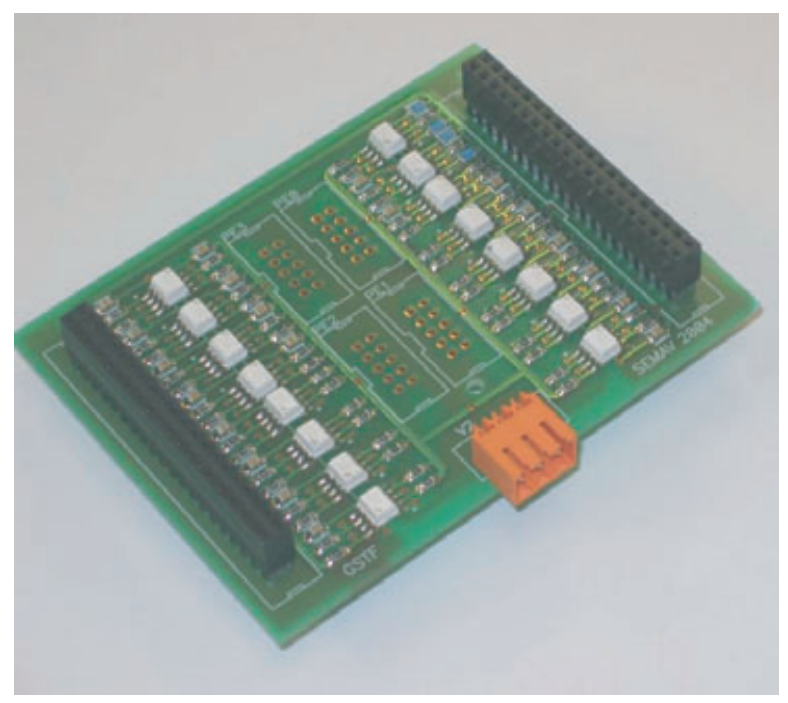

Fig. 5: Photograph of the digital input/output board 


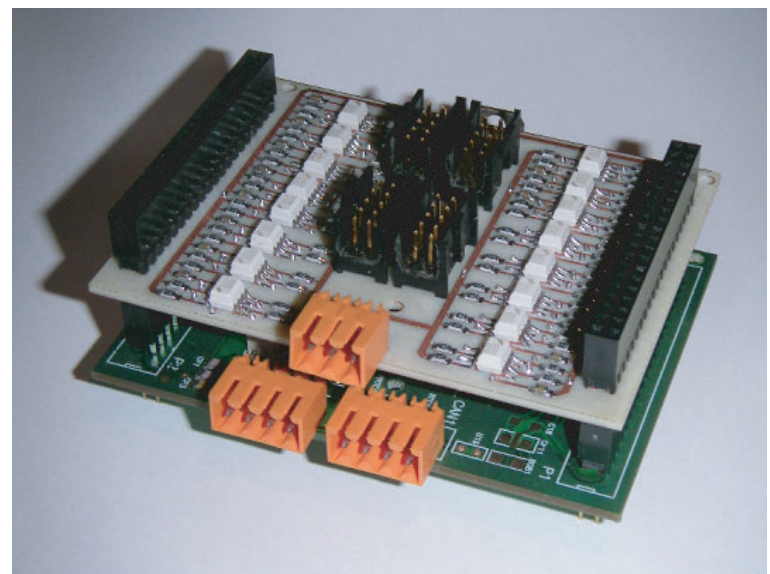

Fig. 6: Photograph of the digital node

figure). In addition an optional power supply connector (V2 in the Fig. 5) can be seen. The chosen optocoupler is the Fairchild MOCD207 (Fairchild Semiconductor Corp., South Portland, ME). It is a dual channel surface mounted optocoupler and offers a typical CTR of $150 \%$ at a very good price. For more details, see the technical specifications. ${ }^{15}$ Figure 6 shows the schemes of the input and output circuitry.

The microcontroller ports work with $3.3 \mathrm{~V}$ but are $5 \mathrm{~V}$ tolerant. As the connectors from the microcontroller module provide $5 \mathrm{~V}$ pins, these can be used as voltage supply for the digital module. The Silicon Laboratories application note AN111 "Using the C8051Fxxx in $5 \mathrm{~V}$ systems"16 provides information and examples for the circuit design. To prevent the microcontroller ports from taking damage, the currents were limited to about $10 \mathrm{~mA}$. This allows enough current to flow on the outputs to drive a LED and the per pin and overall current ratings of the microcontroller are not exceeded. To protect the microcontroller ports from excessive voltages, Zener diodes have been integrated.

Each digital node comprises 20 inputs, four PWM outputs, and eight outputs. The software of the digital node is quite different from analog's software. Additionally to the timedriven loop that samples inputs and sends its state over the network, the digital node must receive messages with the data to put on its outputs. However, no calibration curves or transformations are applied to outgoing or incoming data. Inputs and outputs operations are executed so fast that there is no concern about sampling and updating frequency, easily surpassing $800 \mathrm{~Hz}$. Finally, as an example, Fig. 6 shows a photograph of the digital node. It is composed of the microcontroller board (bottom board) and the digital input/output board (top board). As can be deduced from this photo, if a more complex node is needed, more boards can be stacked to accomplish more functions.

\section{Fieldbus and Communication}

In this section, the communication protocol used in the data acquisition system is explained. The maximum sample rate due to the communication time necessary to broadcast the magnitudes of the simulator-evaluator is obtained and the synchronization solution adopted to sample these magnitudes in partially synchronized way is presented.

\section{Controller Area Network}

Controller Area Network (CAN) is a serial bus standard developed by Robert Bosch GmbH (Stuttgart, Germany) for connecting devices. ${ }^{9}$ It was initially created for the automotive market (as a vehicle bus) but now its applications goes from high-speed networks to low-cost multiplex wiring. Its specification describes the data link layer-comprised of logical link control (LLC) sublayer and the media access control (MAC) sublayer-and some aspects of the physical layer of the ISO/OSI Reference Model. All the other protocol layers are left to the network designer's choice. In the CAN model, all devices are connected to a single shared bus and they are all allowed to start a transmission. Therefore, a carrier sense multiple access/collision avoidance (CSMA/CA) scheme is implemented, that is, if two or more devices start transmitting at the same time, there is a priority-based arbitration scheme to decide which one will be granted permission to continue transmitting. It is a very robust protocol, with error detection and signaling, self-checking, and fault confinement. Bitrates up to $1 \mathrm{Mbit} / \mathrm{s}$ can be used.

There are currently two message formats: the standard format with 11 identifier bits and the extended format with 29 identifier bits, as defined in the CAN Specification version 2.0. ${ }^{9}$ Current version of the acquisition system with a CAN baudrate of $500 \mathrm{Kbps}$ (the baudrate chosen in the system due to a constraint between bus length and speed), a 276.4$\mathrm{Hz}$ sampling rate is achieved. This sampling rate is much more than the necessary and therefore can be considered as sufficient. ${ }^{17}$ Human reaction usually takes more than $0.1 \mathrm{~s}$, so more nodes can be added in the system providing a good sampling rate to measure human reactions. ${ }^{17}$

\section{Evaluating a Driver}

The process of evaluating a driver is divided in to two parts. First, a quantitative evaluation, and second, a qualitative evaluation.

The quantitative evaluation attempts to determine if the driver is able to act on the car controls-pedal, steering wheel, or the adaptation of the disabled driver uses-with the strength and speed needed to drive safely. In addition, reaction time to events has to be measured. In this evaluation, there is no traffic simulation. Visual and audio systems are used to show information or events to the driver. For example, the driver has to press the accelerator until he/she listens to an audible signal, releasing it and braking as fast as possible. There are several different tests, which include visual and sound events, combining two or more car controls, and measurement of strength, speed, and driver's response time. To accomplish these tests, the system operator uses the software simulator but not with full characteristics. The visual and audible functions are reduced to send signal to the driver. Some tests include a single road or traffic signals, but most of them only use lights to indicate the drive when to carry out an action. Also, the acquisition distribution system is used in different ways. 
If for a concrete test some car controls or sensors are not needed - if only maximum pressure over the brake pedal is measured - the nodes related to these devices are configured to not operate, and they do not send data. This allows to increase the sampling frequency of the used nodes that are being used because there is more bandwidth available in the CAN network.

The qualitative evaluation of drivers allows to know if drivers are able to control the car in a very real environment. In this case, all the systems of the simulator are operating: audio and video outputs with public roads simulations, analog and digital inputs from sensors attached to car controls, and all outputs to simulate different events. The system operator shows the driver different scenarios, including different traffic levels and some risky situations. Of course, the objective of this kind of test is not to decide if the attitude of the driver is right and well-mannered, but to decide if the driver's abilities operating car controls and adaptations are enough to drive safety.

\section{CONCLUSIONS AND FUTURE WORK}

Selection of adaptations for disabled drivers needs the evaluation of each one of them through a car simulator/evaluator, to better justify the appropriate device selection. The car simulator needs a data acquisition system to obtain objective measures of the driving results of adaptations for each particular driver. As car simulators/evaluators become more realistic, the data acquisition system must obtain a lot of magnitudes, and it must be able to work over a lot of actuators. Hence, the data acquisition will determine the main features of the car simulator/evaluator. As the number of sensors grows, a real-time data acquisition system must be able to communicate all measures in a deterministic way and in a causal manner. The data acquisition system must be constructed under the architecture of a real-time distributed system. In this sense, this paper has shown an acquisition system based on an inexpensive microcontroller interconnected through a CAN network. This system is able to communicate all messages involved in the driving simulation/evaluation and to process them in a causal manner. Nowadays, the presented acquisition system has been applied to a car simulator with a dynamic platform that gives real sensations to the driver under test. More nodes to actuate over motors of the dynamic platform are being developed. Thus, the real-time data acquisition system has been designed to have enough flexibility, scalability, composability, and network capability to add more nodes with new functionalities.

\section{ACKNOWLEDGEMENTS}

This research work has been supported by the Spanish Research Council (CiCYT) through contract TIN2006-08234.

\section{References}

1. Derriks, H.M., and Mak, P.M., "Underreporting of Road Traffic Casualties. IRTAD Special Report," Ministry of Transport, Public Works and Water Management, The Netherlands (2007).

2. SafetyNet, "Annual Statistical Report 2007. Building the European Road Safety Observatory. Workpackage 1-Task 3," Deliverable No: D 1.16, CARE/EC Project, KfV, NTUA, SWOV, TRL.

3. ECMT, "Public Transport by Bus," European Conference of Ministers of Transport, OCDE Publication Service, Paris, France (1989).

4. Spanish National Statistical Institute, "Survey on Disabilities, Deficiencies and Health Status," URL http://www.ine.es/jaxi/menu.do?type=pcaxis\&path=\%2Ft15/p418\& file $=$ inebase $\& \mathrm{~L}=0$ [accessed 27 July 2009] .

5. Spanish Traffic Administration, "Traffic Statistics Report," URL http://www.dgt.es/portal/ [accessed 27 July 2009].

6. Peters, B., Monitoring and Assessment by Drivers with Special Needs, Simulator Experiences. Vehicle Performance. Understanding Human Monitoring and Assessment, Swets and Zeitlinger Publishers, Leiden, The Netherlands (1999) pp. 24-45.

7. Gregersen, N.P., Falkmer, T., Dols, J., and Pardo, J., "D.4.1, Driving Simulator Scenarios and Requirements. TRAINER Project Deliverable, 1999," Competitive and Sustainable Growth Programme, European Community, Brussels, Belgium (1999).

8. Reed, M.P., and Green, P.A., "Comparison of Driving Performance On-road and in a Low-Cost Simulator Using a Concurrent Telephone Dialing Task," Ergonomics 42(8):1015-1037 (1999).

9. Controller Area Network (C.A.N) Specification, Version 2.0 software, Robert Bosch GmbH, Stuttgart, Germany.

10. Atmel Microcontrollers, Web site: http://www.atmel.com [accessed 27 July 2009].

11. Silicon Laboratories, Data Sheet C8051F040/1/2/3, Revision 1.3., November 2003.

12. Burr-Brown, "FET-Input, Low Power Instrumentation Amplifier INA 121," http://www.burr-brown.com/ [accessed 27 July 2009].

13. Burr-Brown, "Single Supply, MicroPower Instrumentation, Amplifier INA122," URL http://www.burr-brown.com/ [accessed 27 July 2009].

14. Shaw, A.C., "Reasoning About Time in Higher-Level Language Software," IEEE Transactions on Software Engineering 15(7):875-889 (1989).

15. Fairchild Semiconductor, "MOCD207/8 Dual Channel Small Outline Optoisolators, 2000," URL http://www.fairchildsemi.com [accessed 27 July 2009].

16. Silicon Laboratories, Application Note AN111, Using the C8051Fxxx in 5 V Systems, Revision 1.2, December 2003.

17. Green, M., "How Long Does It Take to Stop? Methodological Analysis of Driver Perception-Brake Times," Transportation Human Factors 2(3):195-216 (2000). 